\title{
Glyceollins, a novel class of soybean phytoalexins, inhibit SCF-induced melanogenesis through attenuation of SCF/c-kit downstream signaling pathways
}

\author{
Sun-Hye Shin ${ }^{1}$ and You-Mie Lee ${ }^{1,2}$
}

The anti-melanogenesis effect of glyceollins was examined by melanin synthesis, tyrosinase activity assay in zebrafish embryos and in B16F10 melanoma cells. When developing zebrafish embryos were treated with glyceollins, pigmentation of the embryos, melanin synthesis and tyrosinase activity were all decreased compared with control zebrafish embryos. In situ expression of a pigment cell-specific gene, Sox10, was dramatically decreased by glyceollin treatment in the neural tubes of the trunk region of the embryos. Stem cell factor (SCF)/c-kit signaling pathways as well as expression of microphthalmia-associated transcription factor (MITF) were determined by western blot analysis. Glyceollins inhibited melanin synthesis, as well as the expression and activity of tyrosinase induced by SCF, in a dose-dependent manner in B16F10 melanoma cells. Pretreatment of B16F10 cells with glyceollins dose-dependently inhibited SCF-induced c-kit and Akt phosphorylation. Glyceollins significantly impaired the expression and activity of MITF. An additional inhibitory function of glyceollins was to effectively downregulate intracellular cyclic AMP levels stimulated by SCF in B16F10 cells. Glyceollins have a depigmentation/whitening activity in vitro and in vivo, and that this effect may be due to the inhibition of SCF-induced c-kit and tyrosinase activity through the blockade of downstream signaling pathway.

Experimental \& Molecular Medicine (2013) 45, e17; doi:10.1038/emm.2013.20; published online 5 April 2013

Keywords: c-kit; cyclic AMP; glyceollins; melanogenesis; signaling pathways; stem cell factor

\section{INTRODUCTION}

Melanin is a dark pigment that has an essential role in protection against ultraviolet radiation, and its production is restricted to $\sim 5 \%$ of skin cells that have a common embryological origin and a unique cell type: melanocytes. Melanocytes differentiate from neural crest cells in the neural tubes during the early embryonic stage. ${ }^{1}$ Many cytokines that influence survival, proliferation, differentiation and function have been identified in melanocytes. ${ }^{2}$ Besides their gross phenotypic reaction, the response of melanocytes to cytokines is in general poorly understood. However, the best studied paracrine factor with actions on melanocytes is probably stem cell factor $(\mathrm{SCF}){ }^{3}$

SCF and a kit receptor, c-kit (also known as SCF receptor, or SCFR), are encoded by the steel $(S l)$ locus and the dominant white spotting $(W)$ locus, respectively. SCF/c-kit signaling is known to be involved in melanocyte development during embryogenesis by the analysis of $S l$ and $W$ knockout mice. Soluble form of kit, s-kit, diminishes SCF-induced melanogenesis in human melanocytes. ${ }^{4}$ Several lines of evidence demonstrate the role of SCF/c-kit signaling in the regulation of epidermal melanogenesis under homeostatic, stimulatory or pathogenic conditions, including ultraviolet $\mathrm{B}$ exposure and pigmentation disorders. ${ }^{5,6}$ In the epidermis, keratinocytes produce increased amounts of SCF in response to several stimuli including aging. ${ }^{7}$ Binding of SCF to the extracellular domain of c-kit, a tyrosine kinase receptor, induces dimerization of the receptor. Receptor dimerization is followed by autophosphorylation, with the subsequent activation of a downstream signaling cascade. This cascade involves the stimulation of phosphatidylinositol $3^{\prime}$-kinase and Ras-mitogen-activated protein kinase (Ras-MAPK) through

\footnotetext{
${ }^{1}$ School of Life Sciences and Biotechnology, College of Natural Sciences, Daegu, Republic of Korea and ${ }^{2}$ Research Institute of Pharmaceutical Sciences, College of Pharmacy, Kyungpook National University, Daegu, Republic of Korea

Correspondence: Professor Y-M Lee, Research Institute of Pharmaceutical Sciences, College of Pharmacy, Kyungpook National University, 80 Daehak-ro, Buk-gu, Daegu 702-701, Republic of Korea.

E-mail: lym@knu.ac.kr
}

Received 7 October 2012; revised 11 December 2012; accepted 26 December 2012 
the Shc and Grb2 adaptor proteins, as well as the guanine nucleotide exchange factor, SOS. The end point of this cascade is the activation of the tyrosinase enzyme. Tyrosinase is in turn required for melanocyte survival and melanin biosynthesis. ${ }^{8-10}$

The MAPKs, including extracellular responsive kinase (ERK) and p38 MAPK signaling cascade have been suggested as the signaling pathways modulating melanogenesis. ${ }^{11-13}$ Activation of p38 MAPK positively modulated melanin synthesis ${ }^{12,14}$ by activating cyclic AMP (cAMP) response element-binding protein (CREB), which in turn activates microphthalmiaassociated transcription factor (MITF) expression), a critical melanocyte differentiation and survival transcription factor. ${ }^{15}$ The c-kit receptor also phosphorylates itself through the MAPK pathway. ${ }^{16,17}$ This suggests that protein kinase A signaling is also involved in SCF-induced melanogenesis. Protein kinase A is activated by the elevated cellular cAMP, which leads to the activation of MITF through the activation of CREB, resulting in the expression of tyrosinase, tyrosinase-related protein 1 (TRP-1) and TRP-2 genes. ${ }^{18,19}$ As noted above, it has been shown that SCF induces tyrosinase, ${ }^{20}$ which catalyzes the first two steps of the biosynthesis of eumelanin or pheomelanin, that is, the hydroxylation of tyrosine to dihydroxyphenylalanine (DOPA) and then the oxidation of DOPA to DOPA-quinone. ${ }^{21}$ For this reason, we studied the role of putative inhibitors of $\mathrm{SCF} / \mathrm{c}$-kit signaling in cultured melanoma cells, which provide an in vitro model for melanocytes, and in a zebrafish animal model, as detailed below.

This study used zebrafish as an experimental animal model in a phenotype-based screening for pigmentary inhibitors for the following reasons. The zebrafish system has several advantages, such as numerous quantities of embryos relative to other vertebrates, inducible spawning by light, convenience in observing melanin development, a rapid pigmentation process and high permeability to small molecules. In addition, it possesses epidermal melanocyte equivalents that have similar structural and functional characteristics to those of mammals. ${ }^{22}$ The characteristic external pigment pattern of the zebrafish is generated by an array of three types of pigment cells, all of which are derived from the neural crest. These include melanophores (melanin-containing melanocytes), xanthophores (containing yellow pigment) and iridophores (containing reflecting platelets). ${ }^{23}$ The combination of xanthophores and iridophores leads to the yellow-silver interstripes of the zebrafish, while the melanophores contribute to the longitudinal dark stripes of the epidermis. ${ }^{24,25}$

Glyceollins are a group of phytoalexins that are produced by the soybean plant (Glycine max) under the conditions of stress $^{26,27}$ and abundant in fermented traditional Korean soybean product, such as Chungkookjang, Meju, ${ }^{28}$ which have been used for soothing skin burn or damage traditionally. Glyceollins (a mixture of glyceollin I, II and III) exert antimicrobial activity against several plant pathogens. ${ }^{29,30}$ Moreover, glyceollins function as antiestrogenic agents and may be used for the prevention or treatment of prostate, breast and ovarian carcinomas. ${ }^{31,32}$ The biological functions of glyceollins include anticontractile activity in vascular smooth muscle through the RhoA/Rho kinase signaling pathway, ${ }^{33}$ increased insulin sensitivity ${ }^{34}$ and antimelanin synthesis activity through the inhibition of cAMP in vitro. ${ }^{35}$ However, the biological activity of glyceollins and their underlying mechanisms of action in regard to SCF-induced pigmentation in vitro and in vivo are largely unknown.

In the present study, we isolated glyceollins from elicited soybeans and evaluated the inhibitory activity of glyceollins against SCF-induced tyrosinase activity, MITF expression and cAMP production in B16F10 melanoma cells. Glyceollins effectively suppressed SCF-induced signaling pathways in B16F10 cells. We further examined whether glyceollins could inhibit the skin pigmentation in the zebrafish system through the inhibition of tyrosinase activity. Sox10 expression, a neural crest marker and a key transcription factor that induces MITF gene expression during the differentiation of melanocytes from precursor cells, ${ }^{36}$ was clearly diminished in zebrafish trunk neural tubes by glyceollin treatment. Thus, the results suggest that glyceollins have a strong depigmentation effect in vitro and in vivo and function by inhibiting the SCF-mediated pathway. As such, they could be potential therapeutic agents for the treatment of post-inflammatory hyperpigmentation or skin-whitening agents for cosmetic use.

\section{MATERIALS AND METHODS}

\section{Cell culture and reagents}

B16F10 melanoma cells were cultured on tissue culture plates in Dulbecco modified Eagle's medium (Hyclone, Logan, UT, USA) supplemented with 10\% fetal bovine serum (Hyclone, Logan, UT, USA) and $1 \mathrm{X}$ antibiotics $\left(100 \mathrm{U} \mathrm{ml}^{-1}\right.$ penicillin, $100 \mu \mathrm{g} \mathrm{ml}^{-1}$ streptomycin, all from Invitrogen, Carlsbad, CA, USA) at $37^{\circ} \mathrm{C}$ in a humidified atmosphere incubator containing $5 \% \mathrm{CO}_{2}$. Stock solutions of SCF (Peprotech, Rocky Hills, NJ, USA) and glyceollins were prepared at 1,5 and $10 \mu \mathrm{m}$ in Dulbecco modified Eagle's medium. Antibodies against c-kit, phosphorylated c-kit, ERK and phosphorylated-ERK were purchased from Cell Signaling Technology (Beverly, MA, USA), and antibodies against p38, phosphorylated-p38 and tyrosinase were purchased from Santa Cruz (Santa Cruz, CA, USA).

\section{Isolation of glyceollins}

Glyceollins were isolated using a procedure developed by Boué et al. ${ }^{37}$ Briefly, soybean seeds were scarified and inoculated with Aspergillus sojae. After 3 days, the glyceollins were extracted from the seeds with $80 \%$ ethanol and then isolated using high-performance liquid chromatography (PerkinElmer series 200, PerkinElmer, Waltham, MA, USA). The chemical structures for glyceollin I, II and III are shown in Figure 1.

\section{Zebrafish growth conditions and chemical treatment}

Standard $\mathrm{AB}$ strain zebrafish (Danio rerio) and hemizygous transgenic $\mathrm{Tg}$ (flk-1:EGFP) embryos were maintained at $28^{\circ} \mathrm{C}$ in an oxygenated fish tank on a $14 / 10 \mathrm{~h}$ (light/dark) cycle as previously described. ${ }^{38}$ Embryos were collected from natural matings, dechorionated with pronase at 24, 36 and $48 \mathrm{~h}$ post fertilization (h.p.f.) developmental stages, and maintained. ${ }^{39}$ Bud stage zebrafish embryos were treated with glyceollins at 1,5 and $10 \mu \mathrm{m}$ that were added to the tank water for $40 \mathrm{~h}$. The developmental phenotypes of the embryos were observed with a Zeiss Imager Z1, Zeiss Axioskop (Zeiss, 


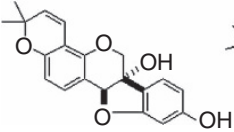

Glyceollin I : 77.96\%

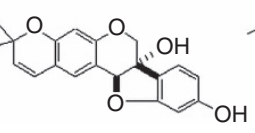

Glyceollin II : $10.07 \%$

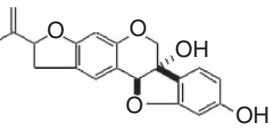

Glyceollin III : 11.97\%

b

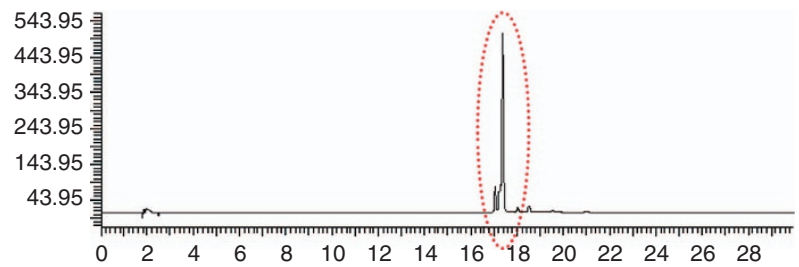

Figure 1 Chemical structure of glyceollins, and high-performance liquid chromatography (HPLC) chromatogram. (a) Chemical structures of glyceollins I, II and III, and their ratio in the mixture employed in this study. b) Glyceollins were isolated from soybean seeds as described in the Materials and Methods section and isolated by HPLC.

Oberkochen, Germany), a Zeiss Stemi 2000 (Zeiss) and a Leica MZFL III (Leica, Welzlar, Germany).

\section{Determination of melanin content}

B16F10 melanoma cells were cultured at $1 \times 10^{5}$ cells per well with $50 \mathrm{ng} \mathrm{ml}^{-1} \mathrm{SCF}$ in the presence or absence of various concentrations of glyceollins $(1,5$ and $10 \mu \mathrm{M})$ for $48 \mathrm{~h}$ in $60-\mathrm{mm}$ dishes. After washing with phosphate-buffered saline, the cells were harvested by trypsinization. The cell pellet was solubilized in $1 \mathrm{~N} \mathrm{NaOH}(100 \mu \mathrm{l})$ containing $10 \%$ dimethylsulfoxide at $80^{\circ} \mathrm{C}$ for $1 \mathrm{~h}$. The absorbance was measured at $450 \mathrm{~nm}$ using a spectrophotometer (Mecasys, Daejeon, South Korea).

\section{Tyrosinase activity}

Glyceollin-treated B16F10 melanoma cells were sonicated in a standard protein extraction solution (20 mM Tris ( $\mathrm{pH} 7.5), 150 \mathrm{~mm}$ $\mathrm{NaCl}, 1 \mathrm{~mm}$ EDTA, $1 \%$ Triton $\mathrm{X}-100$ and $1 \mathrm{X}$ protease inhibitor cocktail) supplemented with $1 \mathrm{~mm}$ phenylmethylsulfonyl fluoride, and the lysates were clarified by centrifugation. After quantification, $30 \mu \mathrm{g}$ total proteins were transferred into 96 -well plates, and $2 \mathrm{mg} \mathrm{ml}^{-1}$ L-DOPA in phosphate-buffer solution was added. Independent experiments were performed at least three times. About 100 zebrafish embryos were treated with glyceollins $(1,5$ and $10 \mu \mathrm{M})$ from bud stage to 48 h.p.f. and sonicated in Pro-Prep protein extraction solution. After quantification, total protein $(250 \mu \mathrm{g})$ was added to $2 \mathrm{mg} / \mathrm{ml}^{-1}$ L-DOPA in a phosphate solution and incubated. Absorbance was then measured at $450 \mathrm{~mm}$ using a spectrophotometer. Tyrosinase activity was expressed as a percentage of the control.

\section{Measurement of cAMP concentration}

The cAMP concentration was measured using a cAMP immunoassay kit (Assay Designs, Ann Arbor, CA, USA). Briefly, B16F10 melanoma cells $\left(3 \times 10^{5}\right)$ were lysed in $0.1 \mathrm{M} \mathrm{HCl}$ to inhibit the phosphodiesterase activity. The supernatants were then collected, neutralized and diluted. After neutralization and dilution, a fixed amount of cAMP conjugate was added to compete with cAMP in the cell lysate for sites on a rabbit polyclonal cAMP antibody immobilized on a 96-well plate. Cells were washed, and a substrate solution was added to determine the activity of the bound enzyme. The absorbance was read at $405 \mathrm{~nm}$. The intensity of the color was inversely proportional to the concentration of cAMP in the cell lysate.

\section{Western blot analysis}

Glyceollin-treated cells were incubated in the presence of SCF (50 $\mathrm{ng} \mathrm{ml}^{-1}$, Peprotech, Rocky Hill, NJ, USA) for $4 \mathrm{~min}$ and lysed. Total cell lysates were analyzed by sodium dodecyl sulfate polyacrylamide gel electrophoresis followed by western blotting with the antibodies against the phosphorylated or unphosphorylated form of p42/44-ERK, AKT and p38 MAPK. Westerns were incubated with horseradish peroxidase-conjugated rabbit or mouse immunoglobulins and then developed by the West Pico Chemiluminescent Substrate (Pierce, Woburn, MA, USA) using standard protocols.

\section{Whole-mount in situ hybridization in zebrafish}

Whole-mount in situ hybridization was performed as previously described. ${ }^{40}$ Briefly, dechorionated embryos at specific stages were fixed with $4 \%$ paraformaldehyde in phosphate-buffered saline. After hybridization, the embryos were incubated with antioxigenin-Dig-AP $\mathrm{F}\left(\mathrm{ab}^{\prime}\right)$ (Boehringer, Ingelheim, Germany) and stained with a staining buffer $\left(\mathrm{pH}\right.$ 9.5, 0.1 м Tris- $\mathrm{HCl}, 0.1 \mathrm{M} \mathrm{NaCl}, 0.05 \mathrm{M} \mathrm{MgCl}_{2}, 0.1 \%$ Tween-20) containing BCIP and NBT. Stained embryos were mounted in glycerol and observed under a microscope with a Nomarski differential interference contrast lens (Axioskop, Zeiss).

\section{Statistical analysis}

One-way analysis of variance was performed to assess the significance of differences among the experimental groups. The level of significance was set at ${ }^{\star} P<0.05$ versus control, ${ }^{\#} P<0.05$ versus SCF. Results are represented as the means \pm s.e.m.

\section{RESULTS}

\section{Glyceollins inhibit pigment production in zebrafish}

To investigate the effect of glyceollins on specific animal pigmentation in the development of melanocyte, we utilized zebrafish embryos. In a preliminary experiment, we tested zebrafish embryos for their feasibility as a model system for melanin synthesis. As a positive control, 1-phenyl-2-thiourea (PTU, $0.3 \mathrm{mg} \mathrm{ml}^{-1}$ ), a typical tyrosinase inhibitor, was used. About 200 embryos at the bud stage were treated with each concentration of glyceollins $(1,5$ and $10 \mu \mathrm{M})$. Pigmentation of zebrafish embryos was decreased by glyceollins in a dosedependent manner (Figure 2A). The number of embryos having pigmentation defects increased as the concentration of glyceollins increased (Figure 2B).

To determine whether the effect of depigmentation was reversible, the embryos were washed intensively at 48 h.p.f. after treatment with PTU and glyceollins $(10 \mu \mathrm{M})$. Embryos were further grown so as to observe body pigmentation at 72 h.p.f. As compared with the no-treatment control group, glyceollin and PTU treatment resulted in a marked inhibition of body pigmentation (Figure 2C), although the effect of PTU was more pronounced, because the concentration of PTU was much higher than that of glyceollins. Removal of PTU and glyceollins allowed the transparent zebrafish to recover some, but not all, of their pigmentation. Therefore, the depigmentation effects of glyceollins were maintained at least for some period of time (24-36 h). 

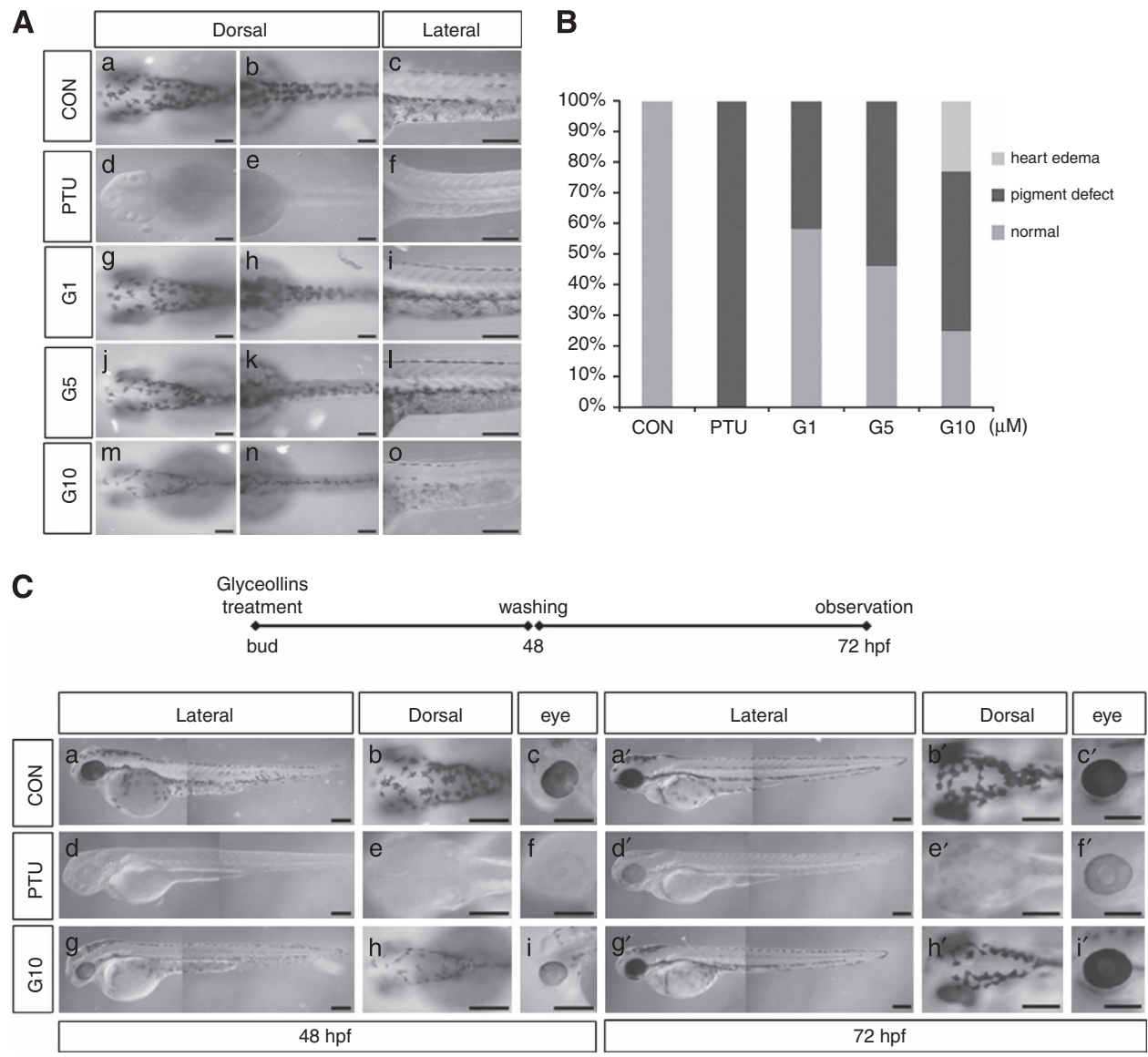

Figure 2 Effect of glyceollins on the pigmentation of developing zebrafish embryos. (A) Live embryos are shown with the head (a, d, g, j, $\mathrm{m}$ ) or the trunk (b, e, h, k, n) or the tail (c, f, i, I, o) from the dorsal and lateral views. Zebrafish were treated with 1-phenyl-2-thiourea (PTU) or glyceollins from the bud stage to $48 \mathrm{~h}$ post fertilization (h.p.f.). (B) Summary of the percentage of each phenotype among 100 embryos per treatment group. (C) Zebrafish embryos at 48 h.p.f. and 72 h.p.f. are shown after treated with PTU and glyceollins. The embryos were treated from bud stage until 48 h.p.f., followed by the removal of the drugs and observation at 72 h.p.f. The eyes are shown in the high-power images.

\section{Glyceollins affect tyrosinase activity and melanin synthesis in zebrafish}

To investigate the molecular targets of glyceollins that inhibit pigmentation in the zebrafish, we determined total melanin content and tyrosinase activity. There were considerable decreases in total melanin content, as well as tyrosinase activity and expression, after treatment with glyceollins (Figures $3 \mathrm{~A}-\mathrm{C})$. As a positive control, we employed PTU and showed that it remarkably decreased melanin content, tyrosinase activity and expression. The inhibitory effects of glyceollins at 1,5 and $10 \mu \mathrm{M}$ on total melanin content and tyrosinase activity were comparable with those of PTU (Figures 3A and B). Their ability at 1 and $5 \mu \mathrm{M}$ to decrease tyrosine expression was less than that of PTU, but glyceollins at a concentration of $10 \mu \mathrm{M}$ showed comparable activity to PTU. These results suggest that glyceollins impair melanogenesis through the inhibition of tyrosinase activity in zebrafish.

\section{Glyceollins inhibit Sox10 expression in zebrafish embryos} In both zebrafish and mice, one transcription factor, Sox10, controls the expression of another, MITF, which in turn regulates a set of genes critical for pigment cell development and pigmentation. Mutations in either Sox10 or MITF impair pigment cell development. ${ }^{41}$ To investigate whether glyceollins affect the differentiation of neural crest cells into melanocytes, we performed whole-mount in situ hybridization with a Sox10 antisense probe in zebrafish embryos. At 24 h.p.f., Sox10 was expressed in melanocyte-sprouting cells in the trunk region (Figure 3D, $\mathrm{a}-\mathrm{a}^{\prime}$ ). However, both PTU (Figure 3D, b- $\mathrm{b}^{\prime}$ ) and the glyceollins (Figure 3D, $\mathrm{c}-\mathrm{c}^{\prime}$ ) attenuated and dispersed the expression of Sox10 at the neural tubes in the trunk region (Figure 3, see the arrows).

\section{Glyceollins inhibit SCF-induced melanogenesis}

To investigate the underlying molecular mechanism to inhibit melanogenesis, we used SCF for induction of melanogenesis and treated $\mathrm{B} 16 \mathrm{~F} 10$ melanoma cells with various concentrations of SCF $\left(5,10,50\right.$ and $\left.100 \mathrm{ng} \mathrm{ml}^{-1}\right)$ for $48 \mathrm{~h}$. SCF increased melanin contents in a dose-dependent manner. The concentration of $50 \mathrm{ng} \mathrm{ml}^{-1}$ SCF induced fairly high melanin content (Figure 4a) and, thus, we used this concentration for further experiments. Next, we investigated whether 


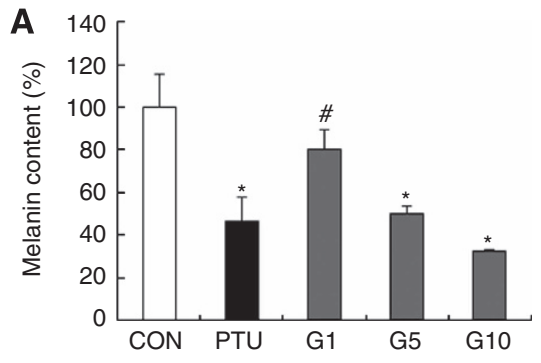

C
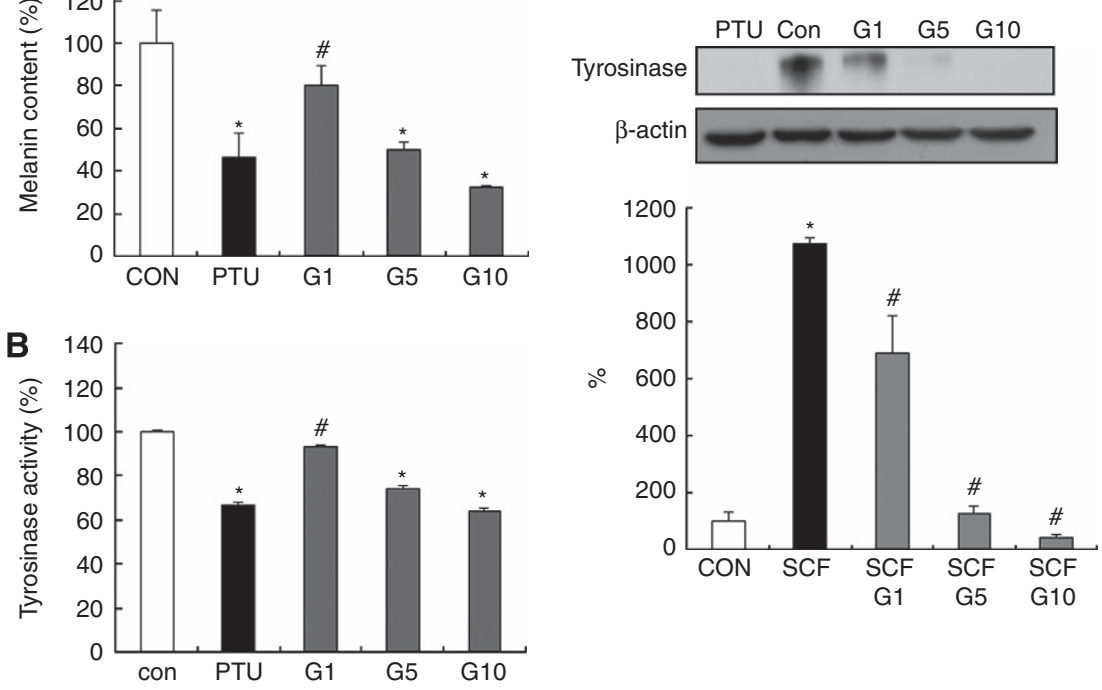

D

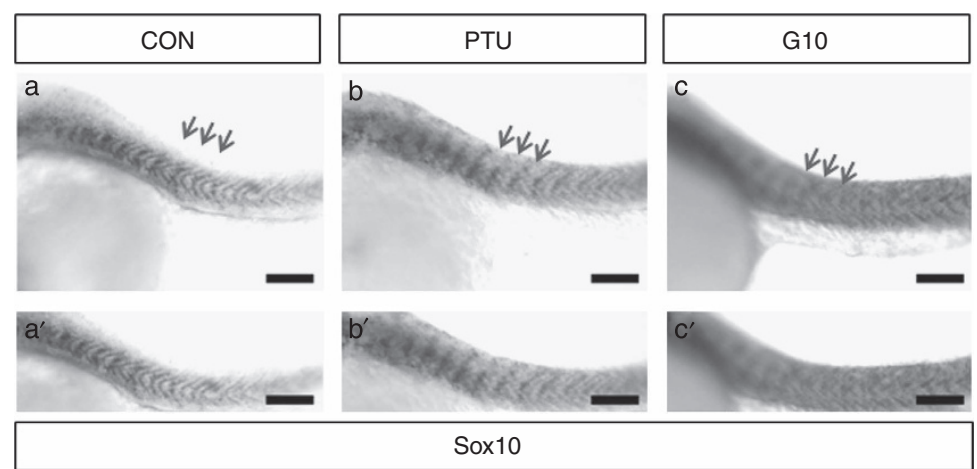

Figure 3 Glyceollins inhibit melanin synthesis through the suppression of tyrosinase activity in zebrafish embryos. (A) About 100 embryos per treatment were dissolved in lysis buffer, melanin pigment was determined using a spectrophotometer at $450 \mathrm{~nm}$. (B) Tyrosinase activity was determined from the melanoma cells and then quantified using a spectrometer. All experiments were repeated three times. (C) The lysate of the zebrafish embryo was subjected to western blot analysis following treatment with stem cell factor (SCF) in the presence or absence of glyceollins. Quantified tyrosinase expression was graphed after normalization to the $\beta$-actin level. * $P<0.05$ versus control; ${ }^{\#} P<0.05$ versus SCF (positive control). (D) Whole-mount in situ hybridization was performed with a Sox 10 antisense probe in each indicated groups. PTU, 1-phenyl-2-thiourea.

glyceollins can inhibit SCF-induced melanin synthesis. Both extracellular, secreted melanin contents and intracellular melanin contents were significantly reduced by the treatment with glyceollins (Figures $4 \mathrm{~b}$ and $\mathrm{c}$ ). Furthermore, the colors of the melanoma cell pellet treated with glyceollins become lighter in a dose-dependent manner compared with SCF-treated positive control cells (Figure $4 \mathrm{~d}$ ). These results indicate that glyceollins inhibited SCF-induced melanin biosynthesis in B16F10 melanoma cells.

\section{Glyceollins inhibit SCF-induced signaling pathways in B16F10 cells}

Treatment of B16F10 melanoma cells with SCF resulted in the phosphorylation of c-kit. Phosphorylation of c-kit was not observed in the absence of SCF, and pretreatment with glyceollins (1, 5 and $10 \mu \mathrm{M})$ significantly inhibited SCFinduced c-kit phosphorylation in a dose-dependent manner (Figure 5a). It is well known that the events downstream of c-kit phosphorylation are related to p44/42 ERK MAPK, and the c-kit/p44/42 ERK MAPK pathway is a common signaling pathway in melanocytes, ${ }^{7,16}$ endothelial cells ${ }^{42}$ and hematopoietic cells. ${ }^{43}$ SCF treatment in melanoma cells induced p44/ 42 ERK phosphorylation, which was inhibited by glyceollins in a dose-dependent manner (Figure 5a). Glyceollins also suppressed SCF-induced AKT and p38-MAPK phosphorylation (Figure 5a). These results suggest that glyceollins successfully suppressed SCF-induced c-kit activation and downstream kinase signaling pathways involved in the function of melanocytes and melanin synthesis. In addition, SCF increased MITF, a melanocyte differentiation factor, ${ }^{16}$ and tyrosinase expression in B16F10 cells. On the other hand, the glyceollins downregulated the SCF-induced expression levels of MITF and tyrosinase (Figures $5 \mathrm{~b}$ and $\mathrm{c}$ ). It has been reported that SCF stimulates tyrosinase activation and melanogenesis, which are mediated by the activation of adenylate cyclase in B16F10 melanoma cells. ${ }^{44}$ Glyceollins significantly suppressed 
a

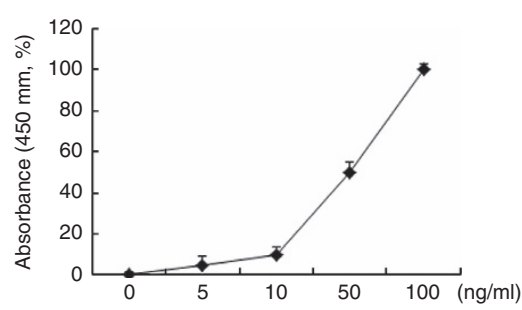

C

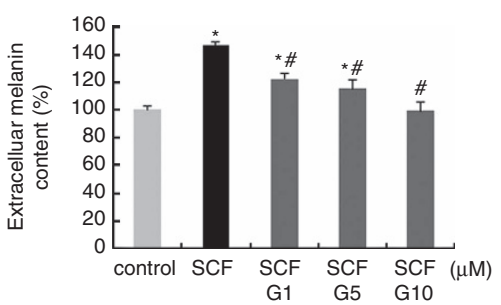

b

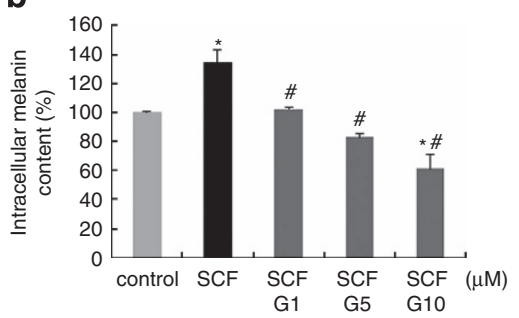

d

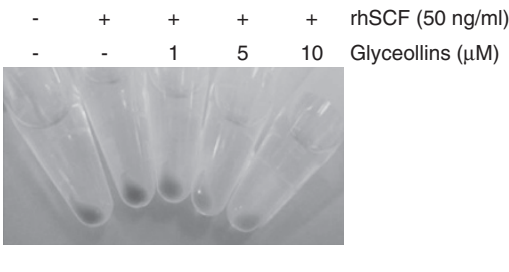

Figure 4 Effect of glyceollins on melanin synthesis and tyrosinase activity in melanoma cells. (a) B16F10 cells were treated with various concentrations of stem cell factor (SCF). Melanin contents were then determined using a spectrophotometer. (b and c) The melanoma cells were incubated with glyceollins ( 1,5 and $10 \mu \mathrm{m})$ in the presence of SCF $\left(50 \mathrm{ngml}^{-1}\right)$ for $48 \mathrm{~h}$, and the extracellular and intracellular melanin contents were detected and quantified using a spectrometer. The results are shown as a percentage of control. All experiments were repeated three times. (d) The image shows the pellet color of glyceollin-treated melanoma cells cultured in vitro. ${ }^{*} P<0.05$ versus control (negative control); ${ }^{\#} P<0.05$ versus SCF (positive control). PTU, 1-phenyl-2-thiourea.

SCF-induced tyrosine's activity (Figure 5d). To investigate whether glyceollins could modulate adenylate cyclase activity, we next measured the intracellular cAMP level stimulated by SCF. Glyceollins did indeed significantly inhibit cAMP production induced by SCF (Figure 5e). Taken together, these data indicate that the depigmentation activity of glyceollins may result from the inhibition of tyrosinase and adenylate cyclase activity involved in the signaling pathway induced by SCF/ckit activation.

Taken together, these results clearly indicate that glyceollins can inhibit melanin biosynthesis induced by SCF. This function is likely to occur via the suppression of c-kit-mediated signaling pathways, including adenylate cyclase and Sox-10 pathways.

\section{DISCUSSION}

Glyceollins are novel phytoalexins derived from soybeans that exist as three isomers. This group of compounds is generated as a plant defense mechanism by an interaction between the soybean plant and many microorganisms. Accordingly, glyceollins exhibit a wide range of toxicities against nematodes, fungi, insects and bacteria for soybean self-protection. ${ }^{30}$ It has also recently been recognized that glyceollins can suppress human breast and ovarian carcinoma tumorigenesis and may modulate potential estrogenic properties in the breast through antiestrogenic effects. ${ }^{32}$ Glyceollins were additionally identified in our earlier study as effective inhibitors of tyrosinase, which was induced in vitro by $\alpha$-melanocyte-stimulating hormone. ${ }^{35}$ SCF is highly associated with melanogenesis by ultravioletirradiated inflammation in keratinocyte $e^{4,45}$ and photoaged pigmentation in fibroblast. ${ }^{46}$ Therefore, we tried to investigate whether glyceollins inhibit SCF-induced melanogenesis.
Here, we provide concrete evidence that glyceollins have a potent inhibitory effect against SCF-stimulated melanogenesis, and we demonstrated their molecular mechanisms of action in B16F10 melanoma cells, as well as in an in vivo zebrafish animal model. We did not show that SCF/c-kit signaling pathway is involved in glyceollins-induced inhibition of zebrafish pigmentation, but administration of anti-c-kit antibody dose-dependently suppressed hair pigmentation in association with decreased melanocyte proliferation and differentiation in mice, ${ }^{47}$ suggesting SCF/c-kit signaling pathway is necessary for the hair or skin pigmentation in mice.

Our current study revealed that glyceollins have an inhibitory effect on melanin content in vitro and in vivo. We confirmed that the intracellular and secreted melanin contents and tyrosinase activity were significantly reduced by glyceollins in melanoma cells. This effect of glyceollins was associated with a significant downregulation of the expression of melanocyte-specific proteins, that is, tyrosinase as well as MITF, compared with the positive control, SCF-treated B16F10 melanoma cells. Because SCF/c-kit is known to enhance the expression of tyrosinase, we hypothesized that the downregulation of SCF/c-kit signaling pathways induced by glyceollins might suppress the expression of tyrosinase. It has been reported that SCF causes tyrosinase activation and melanogenesis through cAMP generation by adenylate cyclase in melanoma cells. ${ }^{48}$ Therefore, inhibition of SCF-stimulated cAMP production in melanoma cells is a key molecular event in the inhibition of melanogenesis.

cAMP generated by the activation of adenylyl cyclase induces CREB to activate MITF gene expression in collaboration with Sox $10 .{ }^{49}$ Therefore, the attenuated expression of MITF caused by glyceollins in this study can likely be 
a

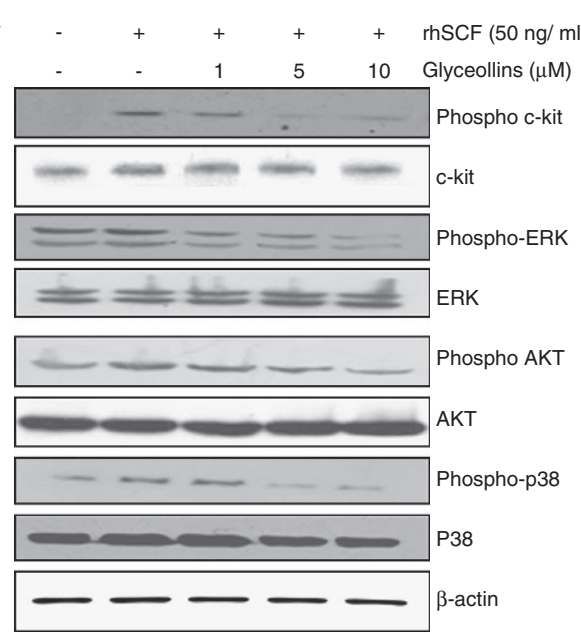

b

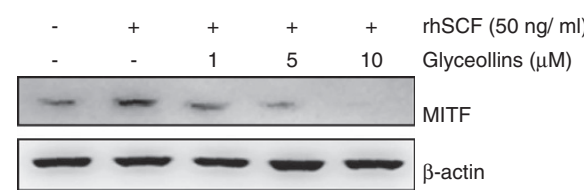

C

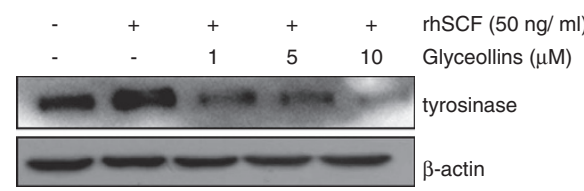

d

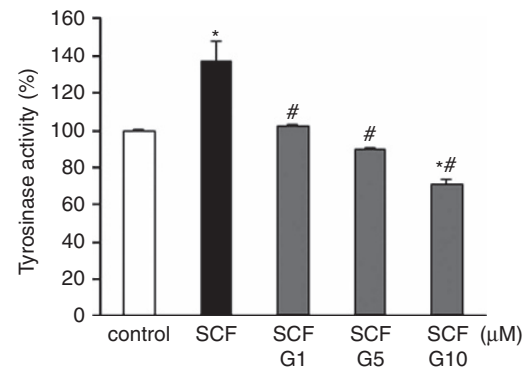

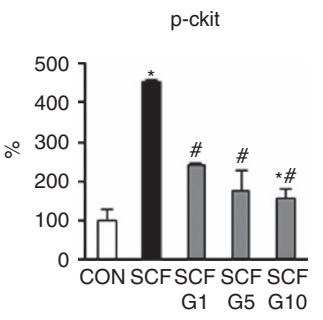
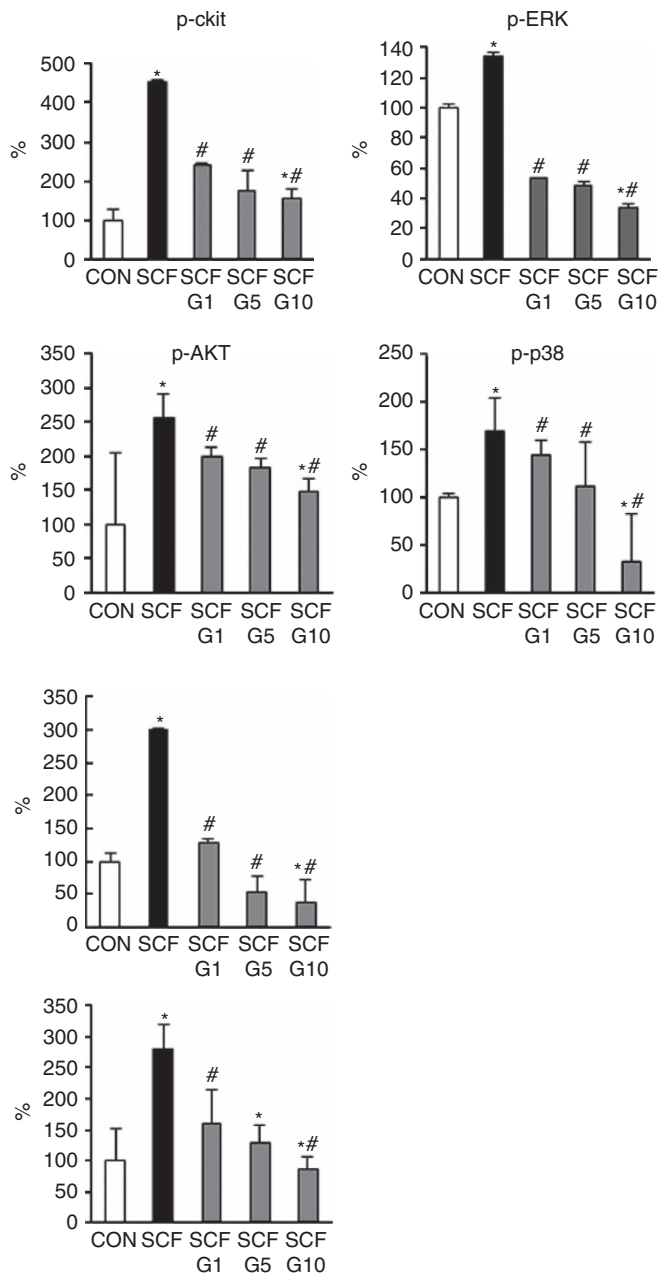

e

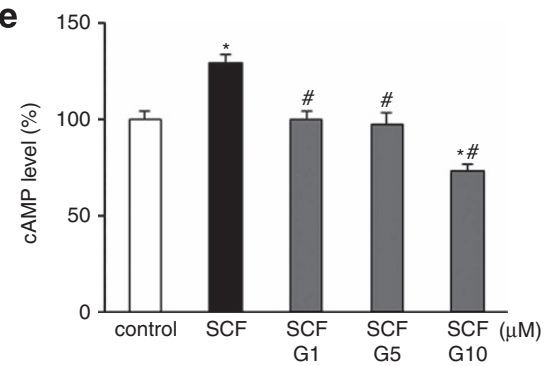

Figure 5 Inhibition of stem cell factor (SCF)-induced c-kit phosphorylation and downstream signaling pathways by glyceollins in B16F10 melanoma cells. (a) Cells were pretreated with glyceollins (1, 5 and $10 \mu \mathrm{m})$ for $48 \mathrm{~h}$, and c-kit phosphorylation was stimulated with recombinant human SCF (rhSCF) $\left(50 \mathrm{ng} \mathrm{ml}^{-1}\right)$ for $4 \mathrm{~min}$. Western blot analysis was performed. (b, c) Melanoma cells were treated with or without glyceollins for $48 \mathrm{~h}$. The microphthalmia-associated transcription factor (MITF) (b) and tyrosinase (c) and $\beta$-actin protein level was determined by western blot analysis. (d) Tyrosinase activity was determined from the melanoma cells incubated with $2 \mathrm{mg} \mathrm{ml}^{-1}$ L-DOPA and then quantified using a spectrometer. (e) The concentration of cAMP was measured using a cAMP assay kit. ${ }^{*} P<0.05$ versus control; ${ }^{P} P<0.05$ versus SCF (positive control). cAMP, cyclic AMP; DOPA, dihydroxyphenylalanine; ERK, extracellular responsive kinase.

attributed to the reduced levels of cAMP and Sox10. MITF expression induced by $\alpha$-melanocyte-stimulating hormone is also inhibited by glyceollins. ${ }^{50}$ As such, glyceollins might also inhibit $\alpha$-melanocyte-stimulating hormone-induced adenylate cyclase activity. In addition, MAPKs such as ERK1/2 and p38 MAPK are also involved in tyrosinase expression in B16F10 cells. ${ }^{51}$ Hence, the inhibition of these MAPKs is an important mechanism by which glyceollins inhibit tyrosinase expression and activity induced by SCF. Because involvement of MAPKs in melanocyte proliferation or differentiation is evidenced only by cell culture system in previous reports and in our study, further investigation whether activity of these MAPKs is inhibited in glyceollins-treated zebrafish embryos should be performed. 
MITF itself is not sufficient to promote increased expression of its target genes. Phosphorylation of MITF serine residue 409 by p90 ribosomal S6 kinase (p90RSK) and serine residue 73 by MAPK1 activated by SCF/c-kit signaling might contribute synergistically to its activation by promoting an interaction with the p300 coactivator. ${ }^{16,17}$ If so, coactivators of the MAPK cascades will thus confer on MITF the ability to contribute significantly to the expression of genes critical for the phenotype and function of the mature melanocyte, such as tyrosinase and TRP $1 .{ }^{52}$

Zebrafish pigmentation and the development of melanocytes derived from neural crest cells were also suppressed by glyceollins, probably through the same mechanism as in B16F10 melanoma cells. It stands to reason that SCF might therefore be the primary stimulator of melanogenesis during embryonic development. For example, Sox10 is expressed in migrating neural crest cells in the trunk neural tubes, which differentiate into sensory neurons in the trunk. ${ }^{53}$ The diminished expression of Sox10 in glyceollin-treated embryos might lead to an ectopic migration path and defected differentiation of neural crest cells. More in detail study using primary neural crest cell culture from embryos homozygous for a Kit null allele, it is shown that the onset of MITF in melanoblast does not require Kit. And treatment of choleratoxin, cAMP activator mimics part of the Kit signaling pathway increases MITF and tyrosine expression in Kit null cells. ${ }^{54}$ This study suggests that MITF is not sufficient for tyrosinase expression in melanoblast and that Kit signaling regulates gene expression during the melanocyte development in vivo. From our results, normal Sox10-induced melanocyte differentiation from neural crest cells likely occurs in collaboration with SCF through increased MITF transcription in the zebrafish. Here we suggest that $\mathrm{SCF} / \mathrm{c}$-kit activates three different downstream pathways, adenylyl cyclase, p38 MAPK and MEK/ERK MAPK pathways. Activation of adenylyl cyclase induces cAMP and protein kinase A to activate CREB, and p38 and ERK MAPK pathways activate MITF. MITF and CREB collaborate to activate tyrosinase and TRP1/2.

\section{CONFLICT OF INTEREST}

The authors declare no conflict of interest.

\section{ACKNOWLEDGEMENTS}

This work was supported by the National Research Foundation of Korea (NRF) grant funded by the Korea government (MEST) (2012028835) and a Small \& Medium Business Administration (SMBA) grant (S1057429).

1 Thomas AJ, Erickson CA. The making of a melanocyte: the specification of melanoblasts from the neural crest. Pigment Cell Melanoma Res 2008; 21: 598-610.

2 Yamaguchi Y, Hearing VJ. Physiological factors that regulate skin pigmentation. Biofactors 2009; 35: 193-199.

3 Longley Jr BJ, Morganroth GS, Tyrrell L, Ding TG, Anderson DM, Williams $\mathrm{DE}$ et al. Altered metabolism of mast-cell growth factor (c-kit ligand) in cutaneous mastocytosis. N Engl J Med 1993; 328: 1302-1307.
4 Kasamatsu S, Hachiya A, Higuchi K, Ohuchi A, Kitahara T, Boissy RE. Production of the soluble form of KIT, s-KIT, abolishes stem cell factorinduced melanogenesis in human melanocytes. J Invest Dermatol 2008; 128: 1763-1772.

5 Hachiya A, Kobayashi A, Ohuchi A, Takema Y, Imokawa G. The paracrine role of stem cell factor/c-kit signaling in the activation of human melanocytes in ultraviolet-B-induced pigmentation. J Invest Dermatol 2001; 116: 578-586.

6 Hattori H, Kawashima M, Ichikawa Y, Imokawa G. The epidermal stem cell factor is over-expressed in lentigo senilis: implication for the mechanism of hyperpigmentation. J Invest Dermatol 2004; 122: 1256-1265.

7 Imokawa G, Kobayasi T, Miyagishi M. Intracellular signaling mechanisms leading to synergistic effects of endothelin-1 and stem cell factor on proliferation of cultured human melanocytes, cross-talk via transactivation of the tyrosine kinase c-kit receptor. J Biol Chem 2000; 275: 33321-33328.

8 Cutler RL, Liu L, Damen JE, Krystal G. Multiple cytokines induce the tyrosine phosphorylation of Shc and its association with Grb2 in hemopoietic cells. J Biol Chem 1993; 268: 21463-21465.

9 Lennartsson J, Blume-Jensen P, Hermanson M, Ponten E, Carlberg M, Ronnstrand L. Phosphorylation of Shc by Src family kinases is necessary for stem cell factor receptor/c-kit mediated activation of the Ras/MAP kinase pathway and c-fos induction. Oncogene 1999; 18: 5546-5553.

10 Liu L, Damen JE, Cutler RL, Krystal G. Multiple cytokines stimulate the binding of a common 145-kilodalton protein to Shc at the Grb2 recognition site of Shc. Mol Cell Biol 1994; 14: 6926-6935.

11 Smalley K, Eisen T. The involvement of p38 mitogen-activated protein kinase in the alpha-melanocyte stimulating hormone (alpha-MSH)-induced melanogenic and anti-proliferative effects in B16 murine melanoma cells. FEBS Lett 2000; 476: 198-202.

12 Hirata $\mathrm{N}$, Naruto $\mathrm{S}$, Ohguchi $\mathrm{K}$, Akao $\mathrm{Y}$, Nozawa $\mathrm{Y}$, linuma $\mathrm{M}$ et al. Mechanism of the melanogenesis stimulation activity of (-)-cubebin in murine B16 melanoma cells. Bioorg Med Chem 2007; 15: 4897-4902.

13 Kim DS, Park SH, Kwon SB, Na JI, Huh CH, Park KC. Additive effects of heat and p38 MAPK inhibitor treatment on melanin synthesis. Arch Pharm Res 2007; 30: 581-586.

14 Singh P, Piotrowski M, Gau AE. Purification and partial characterization of an extracellular melanoprotein from the fungus Venturia inaequalis. $Z$ Naturforsch C 2005; 60: 109-115.

15 Galibert MD, Carreira S, Goding CR. The Usf-1 transcription factor is a novel target for the stress-responsive p38 kinase and mediates UV-induced tyrosinase expression. EMBO J 2001; 20: 5022-5031.

16 Hemesath TJ, Price ER, Takemoto C, Badalian T, Fisher DE. MAP kinase links the transcription factor microphthalmia to c-Kit signalling in melanocytes. Nature 1998; 391: 298-301.

17 Wu MF, Hemesath TJ, Takemoto CM, Horstmann MA, Wells AG, Price ER et al. C-Kit triggers dual phosphorylations, which couple activation and degradation of the essential melanocyte factor Mi. Genes Dev 2000; 14: 301-312.

18 Bertolotto C, Busca R, Abbe P, Bille K, Aberdam E, Ortonne JP et al. Different cis-acting elements are involved in the regulation of TRP1 and TRP2 promoter activities by cyclic AMP: pivotal role of $M$ boxes (GTCATGTGCT) and of microphthalmia. Mol Cell Biol 1998; 18: 694-702.

19 Corre S, Galibert MD. Upstream stimulating factors: highly versatile stressresponsive transcription factors. Pigment Cell Res 2005; 18: 337-348.

20 Vanover JC, Spry ML, Hamilton L, Wakamatsu K, Ito S, D'Orazio JA. Stem cell factor rescues tyrosinase expression and pigmentation in discreet anatomic locations in albino mice. Pigment Cell Melanoma Res 2009; 22: 827-838.

21 Park SH, Kim DS, Lee HK, Kwon SB, Lee S, Ryoo IJ et al. Long-term suppression of tyrosinase by terrein via tyrosinase degradation and its decreased expression. Exp Dermatol 2009; 18: 562-566.

22 Choi TY, Kim JH, Ko DH, Kim CH, Hwang JS, Ahn S et al. Zebrafish as a new model for phenotype-based screening of melanogenic regulatory compounds. Pigment Cell Res 2007; 20: 120-127.

23 Jin EJ, Thibaudeau G. Effects of lithium on pigmentation in the embryonic zebrafish (Brachydanio rerio). Biochim Biophys Acta 1999; 1449: 93-99.

24 Hirata M, Nakamura K, Kondo S. Pigment cell distributions in different tissues of the zebrafish, with special reference to the striped pigment pattern. Dev Dyn 2005; 234: 293-300.

25 Kelsh RN, Brand M, Jiang YJ, Heisenberg CP, Lin S, Haffter P et al. Zebrafish pigmentation mutations and the processes of neural crest development. Development 1996; 123: 369-389. 
26 Graham TL, Kim JE, Graham MY. Role of constitutive isoflavone conjugates in the accumulation of glyceollin in soybean infected with Phytophthora megasperma. Mol Plant Microbe Interact 1990; 3: 157-166.

27 Graham TL, Graham MY. Glyceollin elicitors induce major but distinctly different shifts in isoflavonoid metabolism in proximal and distal soybean cell populations. Mol Plant Microbe Interact 1991; 4: 60-68.

28 Park S, Kim da S, Kim JH, Kim JS, Kim HJ. Glyceollin-containing fermented soybeans improve glucose homeostasis in diabetic mice. Nutrition 2012; 28: 204-211.

29 Daniel O, Meier MS, Schlatter J, Frishknect P. Selected phenolic compounds in cultivated plants:ecologic functions, health implications, and modulation by pesticides. Environ Health Perspect 1999 . 107: 109-114.

30 Bhattacharyya MK, Ward EWB. Resistance, susceptibility and accumulation of glyceollins I-III in soybean organs inoculated with Phytophthora megasperma f. sp. Glycinea. Physiol Mol Plant Pathol 1986; 29: 227-237.

31 Payton-Stewart F, Schoene NW, Kim YS, Burow ME, Cleveland TE, Boue $\mathrm{SM}$ et al. Molecular effects of soy phytoalexin glyceollins in human prostate cancer cells LNCaP. Mol Carcinog 2009; 48: 862-871.

32 Salvo VA, Boue SM, Fonseca JP, Elliott S, Corbitt C, Collins-Burow BM et al. Antiestrogenic glyceollins suppress human breast and ovarian carcinoma tumorigenesis. Clin Cancer Res 2006; 12: 7159-7164.

33 Song MJ, Baek I, Jeon SB, Seo M, Kim YH, Cui S et al. Effects of glyceollin I on vascular contraction in rat aorta. Naunyn Schmiedebergs Arch Pharmacol 2010; 381: 517-528.

34 Boue SM, Carter $\mathrm{CH}$, Ehrlich KC, Cleveland TE. Induction of the soybean phytoalexins coumestrol and glyceollin by Aspergillus. J Agric Food Chem 2000; 48: 2167-2172.

35 Lee Y, Kim H, Lee K, Jeon HW, Cui S, Lee YM et al. Inhibitory effect of glyceollin isolated from soybean against melanogenesis in B16 melanoma cells. BMB Rep 2010; 43: 461-467.

36 Opdecamp K, Nakayama A, Nguyen MT, Hodgkinson CA, Pavan WJ, Arnheiter $\mathrm{H}$. Melanocyte development in vivo and in neural crest cell cultures: crucial dependence on the Mitf basic-helix-loop-helix-zipper transcription factor. Development 1997; 124: 2377-2386.

37 Murohara T, Asahara T, Silver M, Bauters C, Masuda H, Kalka C et al. Nitric oxide synthase modulates angiogenesis in response to tissue ischemia. J Clin Invest 1998; 101: 2567-2578.

38 Westerfield M. The Zebrafish Book. A Guide For Laboratory Use of Zebrafish (Danio rerio). University of Oregon Press: Eugene, 1995.

39 Kimmel CB, Ballard WW, Kimmel SR, Ullmann B, Schilling TF. Stages of embryonic development of the zebrafish. Dev Dyn 1995; 203: 253-310.

40 Jang GH, Ha JH, Huh TL, Lee YM. Effect of proton beam on blood vesse formation in early developing zebrafish (Danio rerio) embryos. Arch Pharm Res 2008; 31: 779-785.

$41 \mathrm{Hou} \mathrm{L}$, Arnheiter H, Pavan WJ. Interspecies difference in the regulation of melanocyte development by SOX10 and MITF. Proc Natl Acad Sci USA 2006; 103: 9081-9085.
42 Blume-Jensen P, Ronnstrand L, Gout I, Waterfield M, Heldin C. Modulation of $\mathrm{Kit} / \mathrm{stem}$ cell factor receptorinduced signaling by protein kinase $\mathrm{C}$. J Biol Chem 1994; 21: 793-802.

43 Heinrich M, Griffith D, Druker B, Wait C, Ott KAZ. Inhibition of c-kit receptor tyrosine kinase activity by STI 571, a selective tyrosine kinase inhibitor. AJ Blood 2000; 96: 925-932.

44 Nakajima H, Fukazawa K, Wakabayashi Y, Wakamatsu K, Imokawa G. Withania somnifera extract attenuates stem cell factor-stimulated pigmentation in human epidermal equivalents through interruption of ERK phosphorylation within melanocytes. J Nat Med 2011; 66: 435-446.

$45 \mathrm{Ha}$ JH, Kang WH, Lee JO, Cho YK, Park SK, Lee SK et al. Clinical evaluation of the depigmenting effect of Glechoma Hederacea extract by topical treatment for 8 weeks on UV-induced pigmentation in Asian skin. Eur J Dermatol 2011; 21: 218-222.

46 Kovacs D, Cardinali G, Aspite N, Cota C, Luzi F, Bellei B et al. Role of fibroblast-derived growth factors in regulating hyperpigmentation of solar lentigo. Br J Dermatol 2010; 163: 1020-1027.

47 Botchkareva NV, Khlgatian M, Longley BJ, Botchkarev VA, Gilchrest BA. $\mathrm{SCF} / \mathrm{c}-\mathrm{kit}$ signaling is required for cyclic regeneration of the hair pigmentation unit. FASEB J 2001; 15: 645-658.

48 Grabbe J, Welker P, Dippel E, Czarnetzki BM. Stem cell factor, a novel cutaneous growth factor for mast cells and melanocytes. Arch Dermatol Res 1994; 287: 78-84.

49 Potterf SB, Mollaaghababa R, Hou L, Southard-Smith EM, Hornyak TJ, Arnheiter $\mathrm{H}$ et al. Analysis of SOX10 function in neural crest-derived melanocyte development: SOX10-dependent transcriptional control of dopachrome tautomerase. Dev Biol 2001; 237: 245-257.

50 Lee YS, Kim HK, Lee KJ, Jeon HW, Cui S, Lee YM et al. Inhibitory effect of glyceollin isolated from soybean against melanogenesis in B16 melanoma cells. BMB Rep 2010; 43: 461-467.

51 Tsang T, Ye Y, Tai WC, Chou GX, Leung AK, Yu ZL et al. Inhibition of the p38 and PKA signaling pathways is associated with the anti-melanogenic activity of Qian-wang-hong-bai-san, a Chinese herbal formula, in B16 cells. J Ethnopharmacol 2012; 141: 622-628.

52 Yasumoto K, Yokoyama K, Takahashi K, Tomita Y, Shibahara S. Functional analysis of microphthalmia-associated transcription factor in pigment cellspecific transcription of the human tyrosinase family genes. J Biol Chem 1997; 272: 503-509.

53 Carney TJ, Dutton KA, Greenhill E, Delfino-Machín M, Dufourcq P, Blader $P$ et al. A direct role for Sox10 in specification of neural crest-derived sensory neurons. Development 2006; 133: 4619-4630.

54 Hou L, Panthier JJ, Arnheiter H. Signaling and transcriptional regulation in the neural crest-derived melanocyte lineage: interactions between KIT and MITF. Development 2000; 127: 5379-5389.

This work is licensed under a Creative Commons Attribution-NonCommercial-NoDerivs 3.0 Unported License. To view a copy of this license, visit http:// creativecommons.org/licenses/by-nc-nd/3.0/ 